\title{
Pentamidine Isethionate
}

National Cancer Institute

\section{Source}

National Cancer Institute. Pentamidine Isethionate. NCI Thesaurus. Code C29349.

A synthetic amidine derivative, Pentamidine Isethionate is an antiprotozoal and antifungal agent that appears to interact with the minor groove of AT-rich DNA regions of the pathogen genome, interfering with DNA replication and function. It is effective in the treatment of trypanosomiasis, leishmaniasis, some fungal infections, and Pneumocystis carinii pneumonia in HIV-infected patients. ( $\mathrm{NCl04)}$ 